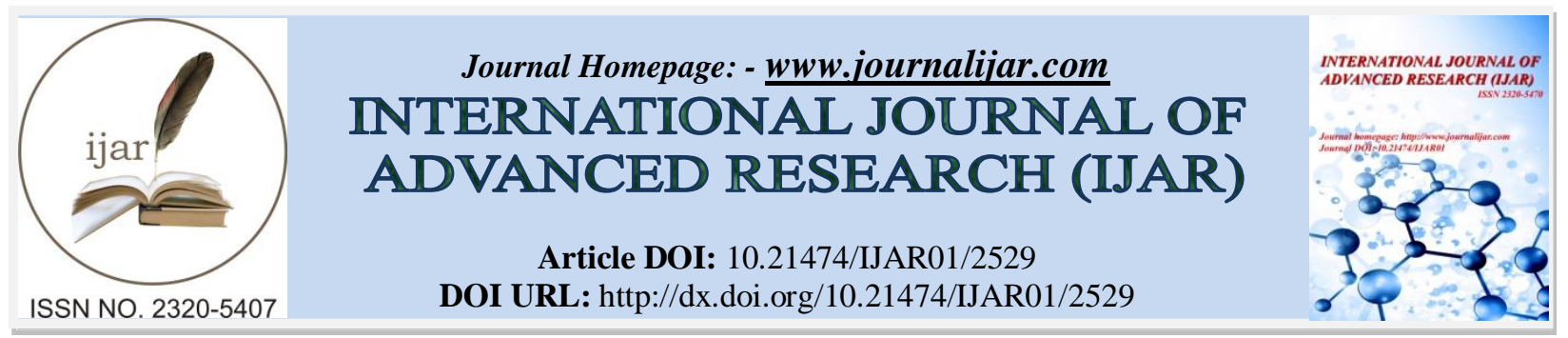

RESEARCH ARTICLE

\title{
ALTERED EXPRESSION OF MIR-214, MIR-3120 AND PTEN IN IRON-INDUCED EXPERIMENTAL EPILEPSY MODEL OF POST-TRAUMATIC EPILEPSY AND THE EFFECT OF CURCUMIN.
}

Prince Kumar, Rameshwar Singh and Deepak Sharma*.

Neurobiology Laboratory, School of Life Sciences, Jawaharlal Nehru University, New Delhi 110067, India.

\section{Manuscript Info}

Manuscript History

Received: 25 October 2016

Final Accepted: 23 November 2016

Published: December 2016

Key words:-

Curcumin, Post-traumatic epilepsy, miRNA, miR-214, miR-3120, Pten.

\section{Abstract}

MicroRNAs are known to be involved in the pathogenesis of epilepsies. The objective of the present study was to investigate the changes in expression of miR-3120, miR-214 and Pten gene in ironinduced experimental epilepsy model of post-traumatic epilepsy. In addition, the role of these miRNAs and Pten was also studied in the antiseizure action of curcumin. The results showed that in ironinduced epileptogenesis, expression of miR-3120 and miR-214 is reduced and Pten gene expression is upregulated. The results suggest that these miRNAs may be involved in the pathogenesis of epilepsy. Curcumin's antiseizure effect is mediated by an increase in the expression of miR-3120 and miR-214 and downregulation of Pten gene expression.

Copy Right, IJAR, 2016,. All rights reserved.

\section{Introduction:-}

MicroRNAs (miRNAs) are endogenous non-coding small RNAs. They regulate gene expression by inhibiting/degrading protein coding mRNAs. However, miRNAs can also upregulate translation (Cao et al., 2006; Kosik, 2006). miRNAs have been found to participate in the molecular mechanisms of many pathophysiological processes including those of neurological disorders. miRNA-214 targets Huntington's gene (Sinha et al., 2011). It is also involved in NMDA receptor-related memory processes (Wang et al., 2014). miRNA-3120 is a brain-specific miRNA which is involved in uncoating of vesicles (Scott et al., 2012) and is also involved in learning. miRNA-214 has been reported to modulate NMDA receptor-mediated neurobehavioral dysfunction (Wang et al., 2014).

Changes in the expression of miRNAs occur in epilepsies as miRNAs target a variety of pathways i.e. inflammation, apoptosis, dendritic growth, and spine dynamics, neurites growth, $\mathrm{Ca}^{2+}$ - calmodulin-dependent protein kinase-II and thus NMDA receptors. miRNAs are thus seem to be involved in the pathogenesis of epilepsies. For example, miRNA-146a was shown to be upregulated in human temporal lobe epilepsy (TLE), miR-219 was found to decrease in kainic acid model of epilepsy and in the CSF of epilepsy patients, and silencing of miR-219 was found to induce seizures (Dogini et al., 2015). Several miRNAs were found altered in a lithium-pilocarpine model of status epilepticus. miRNA-214 was found to be down-regulated in mesial temporal lobe epilepsy patients (Li et al., 2014). Brain-specific miR-219 and 134 were found to be significantly upregulated with seizures (Li et al., 2014), miRNAs have even been considered as biomarkers of epilepsy as different miRNAs may be involved in different epilepsies (Li et al., 2014).

Iron-induced experimental epilepsy in rodents models the human clinical post-traumatic epilepsy (Willmore et al., 1978). This experimental model has often been used to investigate the mechanism of epileptogenesis and 
pharmacology of epilepsy (Willmore, 1990). In iron- induced epileptogenesis, glutamatergic mechanisms have been implicated. For example glutamate transporters are down-regulated, glutamate receptors are upregulated and extracellular glutamate levels increase in iron-induced epileptogenesis (Ueda et al., 2001). In the present study, this model was adopted to further investigate the possible involvement of miRNAs in the pathogenesis of epilepsy as miRNAs may show differential responses in different epilepsies/models (Li et al., 2014), Glutamatergic mechanisms are involved in iron-induced epilepsy (Mishra et al., 2013; Ueda and Willmore, 2000) and miRNAs are involved in the regulation of glutamatergic mechanisms (Harraz et al., 2012; Kawashima et al., 2010; Morel et al., 2013). In the present study, we explored the correlation between miRNA-214, miRNA-3120 and development of epileptiform activity in iron-induced epileptogenesis by investigating their expression profiles in epileptogenic tissue.

In addition, expression status of Pten gene (Backman et al., 2001) during iron-induced epileptogenesis was also studied. Specific disruption of Pten gene is known to result in epileptogenesis (Backman et al., 2001; Meng et al., 2013), and miR-214 and miR-3120 both targets Pten gene (Yang et al., 2008)). Pten epileptogenesis is mediated by PTEN inhibition of mTOR signalling. It is the hyperactivation of mTOR that is epileptogenic (Meng et al., 2013). mTOR pathway has been found to mediate temporal lobe epilepsy (Zeng et al., 2009). Therefore, it is of interest to also investigate the Pten gene expression in epileptogenesis.

The second aim of the present study was to determine whether curcumin's anti-epileptic effect (Jyoti et al., 2009) involves curcumin's action on miRNAs. Curcumin is diferuloylmethane. It is obtained from the rhizome of the plant Curcuma longa and is a common agent used as a spice in Indian food. Curcumin has been termed as a cureamine and experimentally it has been found to have multiple pharmacological and therapeutic properties of possible clinical importance (Satoskar et al., 1986; Strimpakos and Sharma, 2008). Neurologically it has been found to be a neuroprotective agent (Cole et al., 2007), and exerts antiepileptic action in experimental epilepsy (Noor et al., 2012). It significantly attenuates electrographic and behavioral seizures and their biochemical measures in iron-induced experimental epilepsy (Jyoti et al., 2009). Curcumin has been shown to alter expression of several miRNAs i.e. miR103, 140, 146a, 148a, 199a, 21, 22, 204, 98, 7 in a variety of experimental conditions (Sun et al., 2008). In the current study we, therefore investigated whether the antiepileptic action of curcumin is mediated by its action on the expression miRNA-214 and miR-3120 and Pten gene in iron-induced experimental epilepsy. Curcumin appears to be an inhibitor of mTOR signalling (Meng et al., 2013) and therefore curcumin's and pten gene's influence on Pten gene is of interest.

\section{Methods:- \\ Materials:-}

Electrodes and wires used in electrophysiology and stereotaxy were made of stainless steel. Curcumin and chemicals used were obtained from Sigma-Aldrich chemical company USA. Curcumin was mixed with standard diet ingredients at a concentration of $1000 \mathrm{ppm}$ and food pellets were made.

\section{Animal treatment plan:-}

Rats of six to eight months of age (male Wistar) were used for this study. Animals were housed in pairs in standard laboratory cages and maintained at $23 \pm 4^{\circ} \mathrm{C}$, under 12 hour light /12 hour dark cycle. All the experimental protocols were approved by the committee for Purpose of Control and Supervision of Experimental Animals (CPCSEA) and the Institutional Animal Ethical Committee (IAEC) of Jawaharlal Nehru University, New Delhi, India. Animals were grouped as follows. To characterize the epileptiform electrical activity and to ascertain the effect of curcumin on it (in our set of animals), a group of animals $(\mathrm{n}=10)$ were made epileptic by intracortical injection of $\mathrm{FeCl}_{3}$ (procedure described below). In five of these animals, development and progression of epileptiform activity was studied and in the other five animals effect of curcumin was assessed, five animals were used as controls in which intracortical injection of saline was given instead of $\mathrm{FeCl}_{3}$. In these animals, electrocorticographic activity was recorded as in $\mathrm{FeCl}_{3}$-injected animals.

For studying changes in miRNAs, animals were grouped as follows: Group I $(n=12)$ consisted of controls which received an intracortical injection of saline (in place of $\mathrm{FeCl}_{3}$ ) and had electrodes implanted for electrocorticography. These animals served as controls for the next two groups of animals. Group II ( $\mathrm{n}=16$ ) consisted of iron- induced epileptic rats. These rats were made epileptic by intracortical injection of $\mathrm{FeCl}_{3}$ (procedure described below). In these animals, miRNA were estimated at various time points and had electrodes implanted for electrocorticography. Group-III $(n=8)$ animals consisted of iron-induced epileptic rats with electrodes implanted for electrocorticography that were fed curcumin. Curcumin treatment was started on the very day 
intracortical injection of $\mathrm{FeCl}_{3}$ was given. In these animals, miRNAs estimations were done to determine the effect of curcumin.

\section{Surgical procedure and recordings:-}

Electrodes were implanted stereotaxically under anesthesia with $4 \%$ isoflourine.Six burr holes of $0.5 \mathrm{~mm}$ diameter (one for iron injection and other five for epidural electrodes placement) were drilled at the surface of the skull at stereotaxically marked sites. $\mathrm{FeCl}_{3}\left(5 \mu \mathrm{l}\right.$ containing $100 \mathrm{mM} \mathrm{FeCl}_{3}$ dissolved in physiological saline with flow rate of $1 \mu \mathrm{l} / \mathrm{min}$ for $5 \mathrm{mins}$. Coordinates for $\mathrm{FeCl}_{3}$ injection were antero-posterior $=-1.0 \mathrm{~mm}$; lateral $=1.0 \mathrm{~mm}$ and ventral (depth) $=1.5 \mathrm{~mm}$ in somatosensory region of cortex with the help of injector cannula. After injection, burr hole was sealed with bone wax (Mishra et al., 2013). For electrographic recording, four stainless-steel epidural screw electrodes were implanted at the coordinates, $2 \mathrm{~mm}$ posterior and anterior to bregma, and $2 \mathrm{~mm}$ lateral in the somatosensory cortex. One screw electrode was placed on frontal sinus as animal ground. Each electrode was connected with a wire to each individual pin of a 9 pin adaptor. Later, 9 pins adapter was fixed to the surface of the skull using adherent dental acrylic cement to create a stout platform. After surgery, Animals were kept individually and allowed to recover and habituate for 1 week before recordings were started. Operated rats were given proper post-operative care (Jyoti et al., 2009). Curcumin treatment was started on the very day intracortical injection of $\mathrm{FeCl}_{3}$ was given.

After 3 days of post-operative recovery, rats were prepared for electrocorticogram recordings. Recordings were made using Grass polygraph recorder as described previously (Mishra et al., 2013). Electrophysiological recordings were obtained from wake and conscious unrestrained animals and the occurrence of epileptiform electrographic activity was assessed during passive/quite and wakefulness condition. Simultaneous recording of ECoG and multiple unit activity potentials (MUA) was done to verify that there was an epileptic-activity associated increase in neuronal firing. MUA were amplified and filtered $(300 \mathrm{~Hz}$ to $10 \mathrm{KHz})$ by Grass P511J preamplifiers, electronically discriminated using a window discriminator (WPI) and displayed on an oscilloscope. The standard EEG waves and MUA pulses were recorded as described in previous studies (Mishra et al., 2013). MUA potential counts were used to quantify the seizure activity.

\section{RNA isolation and quantification:-}

Rats were sacrificed by cervical dislocation and their brains were removed. The cortex was dissected out. Brain samples were crushed with liquid nitrogen and mixed with Trizol/TRI-Reagent (Sigma-Aldrich) followed by alcohol precipitation of total RNAs including micro-RNA using isopropanol and $70 \%$ ethanol. The palate which was obtained by centrifugation of precipitating RNA was dissolved in RNAase free DEPC-treated water, and the concentration was quantified by using Thermo Scientific Nanodrop system and aliquots were made after equilibrating the concentration of RNA and stored at $-80^{\circ} \mathrm{C}$ for further use (Aronica and Gorter, 2007; Mishra et al., 2013).

Quantification of miR-3120 and miR-214 was done by using Life Biosystems micro RNA assay kit (Scott et al., 2012). cDNA of miRNA-3120 and miR-214 were synthesized and amplification was done using stemloop PCR in which stemploop primers of miR-3120 and miR-214 from Life Biosystems were used to observe expression pattern of both miRNAs. U6sn RNA was used as an endogenous control. Expression of both miRNAs was quantified by using ImageJ software through densitometric analysis of pictures taken from 4\% agarose gels(Scott et al., 2012).

Quantification of Pten was done through two-step semi-quantitative PCR, using Applied Biosystems cDNA synthesis kit and using primers for Pten. GAPDH was used as an endogenous control. Primers for Pten and GAPDH were 5'CAATGTTCAGTGGCGGAACTT3' forward, 5' GGCAATGGCTGAGGGAACT3' reverse and 5'ACCACAGTCCATGCCATCAC3' forward, 5'CACCACCCTGTTGGCTGTAGCC3' reverse. Expression of the gene was quantified through densitometry analysis of pictures of $1 \%$ agarose gels using ImageJ software (Rahal and Simmen, 2010).

\section{Statistical analysis:-}

The results were expressed as mean, \pm SEM. Statistical analysis was performed by using one-way analysis of variance (ANOVA) with post hoc statistical tests. 


\section{Results:-}

Electrographic seizure activity and the effect of curcumin in iron-induced epileptic animals:-

Experimental animals which were given intracortical iron injection, developed epileptiform electrographic activity on their electrocorticograms (ECoG) (Fig.1). Distinct chronic epileptiform activity on ECoG began to appear around day 7 onwards (Fig-1A, a-d). The epileptiform activity was spontaneous and recurrent and consisted of isolated spikes, polyspikes, spike-waves complexes, behavioral seizures activity concomitant with ECoG paroxysms progressed with time and consisted of more facial automatisms, head nods following pauses in behavior, steadfast posture, tonic flexing concurrent with biting and chewing hindlimb extremity. The epileptiform activity was quantified by multiple unit action potentials (MUA). The MUA recordings clearly showed the progressive development and build-up of the epileptic ECoG activity (Fig. 1 B).

Curcumin treatment of epileptic animals for various durations suppressed the epileptiform activity on ECoG (Fig 1. e-h). Statistical comparison of the corresponding MUA counts from controls and curcumin-treated animals (Fig-1B) clearly showed the quantitative extent of the decrements of the epileptic activity after curcumin treatment $\quad\left(\mathrm{F}_{3,36}=\right.$ $159, \mathrm{p}<0.01)$
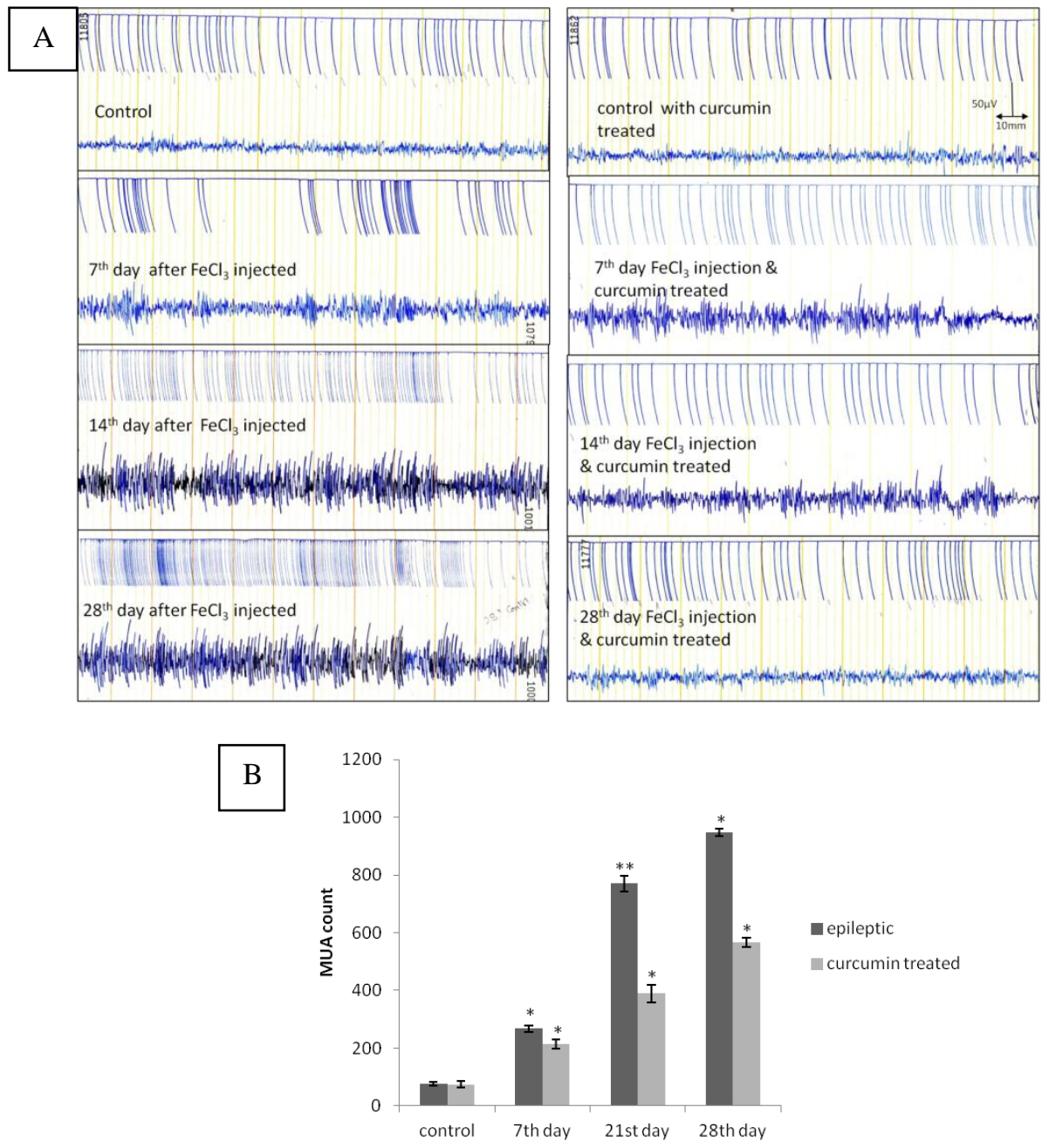

Fig. 1 - (A) Representative sample of polygraph recordings (ECoG and Multiple-unit activity) from somatosensory region of cortex of epileptic and curcumin treated epileptic rat showing epileptogenesis (a) control (b-d), day7,21 28 post-iron injection (e-h) effect of curcumin treatment on epileptogenesis (e)control, (f-h) day7,21 and $28^{\text {th }}$ of curcumin treatment (B) effect of curcumin treatment for 7,21 and 28day on MUA in epileptic rats each bar represents mean SEM of 4 rat. Statistical comparision is with the respective control $(F(3,36)=159), p<0.01)$. *p< $0.05, * * \mathrm{p}<0.01, * * * \mathrm{p}<0.001($ ANOVA) 
Expression of miR-3120 and miR-214 in iron- induced epilepsy:-

Levels of miR-3120 and miR-214 were determined in the cortex of iron-induced epileptic rats at days 7, 14, 21, 28 after induction of epilepsy. Figure-2 shows that the levels of both miR-3120 and miR-214, compared with the controls, decreased with the progression of electrographic seizure activity (Fig.2A \& B) $\left(F_{3,36}=17, p<0.01 ; F_{3,36}=\right.$ $66, \mathrm{p}<0.01$ ), indicating that the expression of these miRNAs falls during the development of iron-induced epileptiform activity.

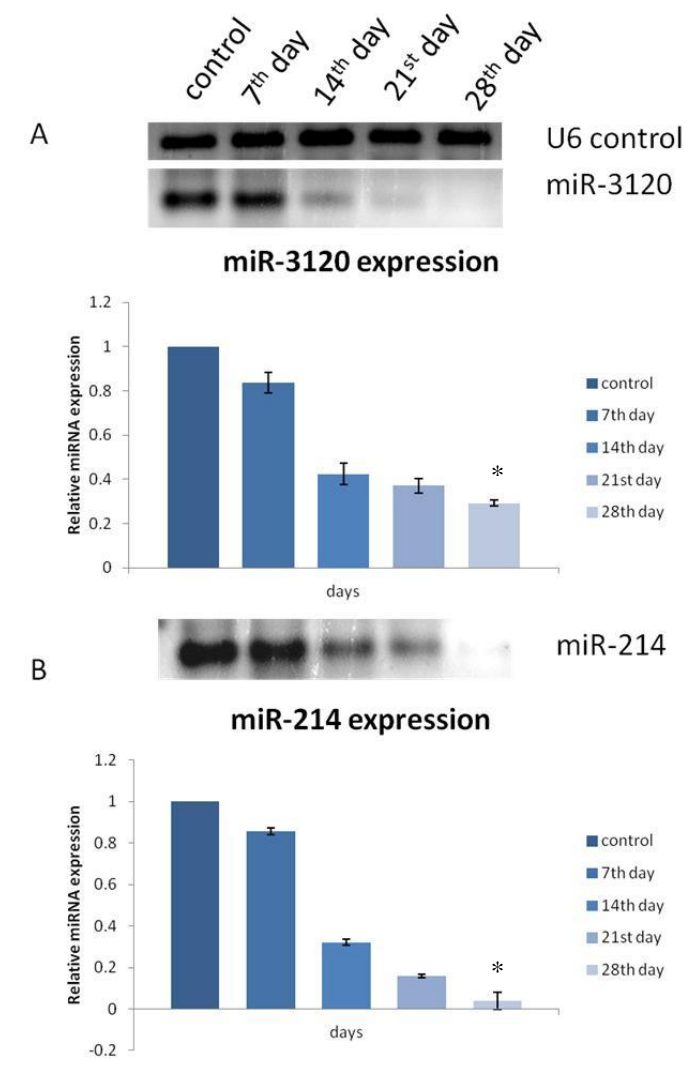

Fig. 2 - Relative miR-3120 (A) and miR-214 (B) expression levels in rat cortex of iron induced epilepsy measured by semi qRT-PCR Each bar represents the mean \pm SD of SEM levels of miRNAs. Statistical comparision of values at day 7, 14, 21, and 28 of Epileptogenesis are with the controls ( $\mathrm{n}=4, \mathrm{p}<0.05$ (ANOVA)) $\&(\mathrm{n}=4, \mathrm{p}<0.05$ (ANOVA)). Electrophoresis bands (agarose gel) of RT-PCR products corresponding to mRNA expression are presented above the bars. Decrease in miRNAs expression with the Epileptogenesis is evident.

Effect of curcumin on the expression of miR-3120 and miR-214 in iron-induced epilepsy:-

Treatment of iron-induced epileptic rats with curcumin partially countered the epileptogenesis-associated decline in miR-3120 levels in their cortex as significantly higher levels of miRNAs were detected at day 28 after curcumin treatment (Fig.3). Seven days treatment did not significantly elevate the level of expression, 28 days treatment, however, resulted in significant elevation of the expression of both miR-3120 and miR-214 $\left(\mathrm{F}_{2,8}=18, \mathrm{p}<0.01 ; \mathrm{F}_{2,8}\right.$ $\mathrm{p}<0.05$ ) (Fig. 4). Thus, curcumin treatment partially prevented the epileptogenesis associated decline in the levels of both miRNAs (Fig. 4). 


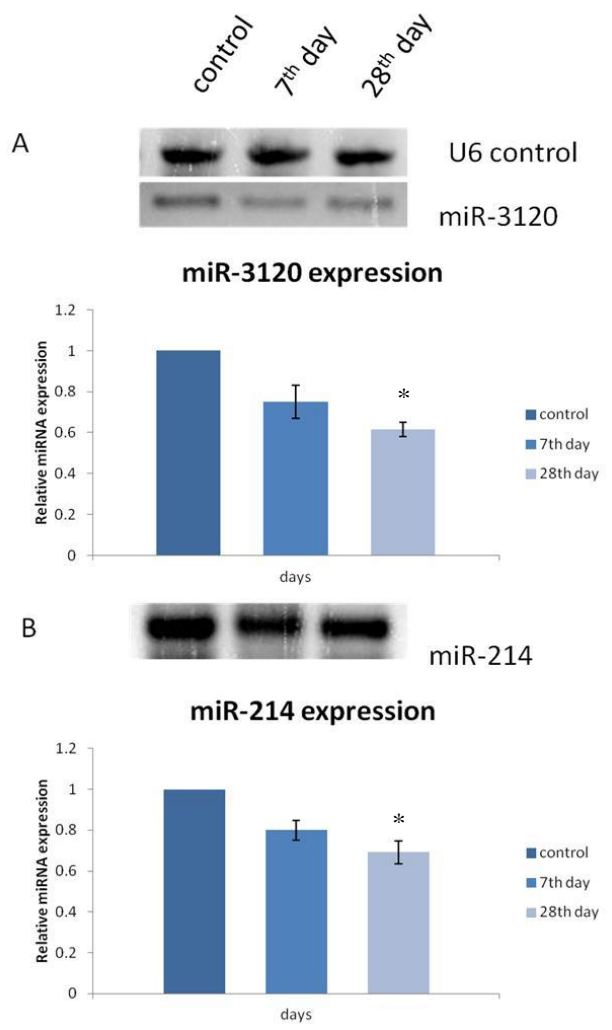

Fig 3. Effect of curcumin on relative miR-3120 (A) and miR-214 (B) expression in rat cortex of iron-induced epileptic rats measures by semi qPCR. Each bar represents the mean \pm SD of SEM levels of miRNAs. Statistical comparison of values at day 7 and $28^{\text {th }}$ of epleptogenesis are with the control. Electrophoresis bands (agarose gel) of RT-PCR products corresponding to mRNA expression are presented above the bars $n=3, p<0.05$ (ANOVA)) \&. n=3, p< 0.05 (ANOVA)). Increase in levels of expression (comparison of values at day 7 day 28 with those of Fig- 4 after curcumin treatment are evident.

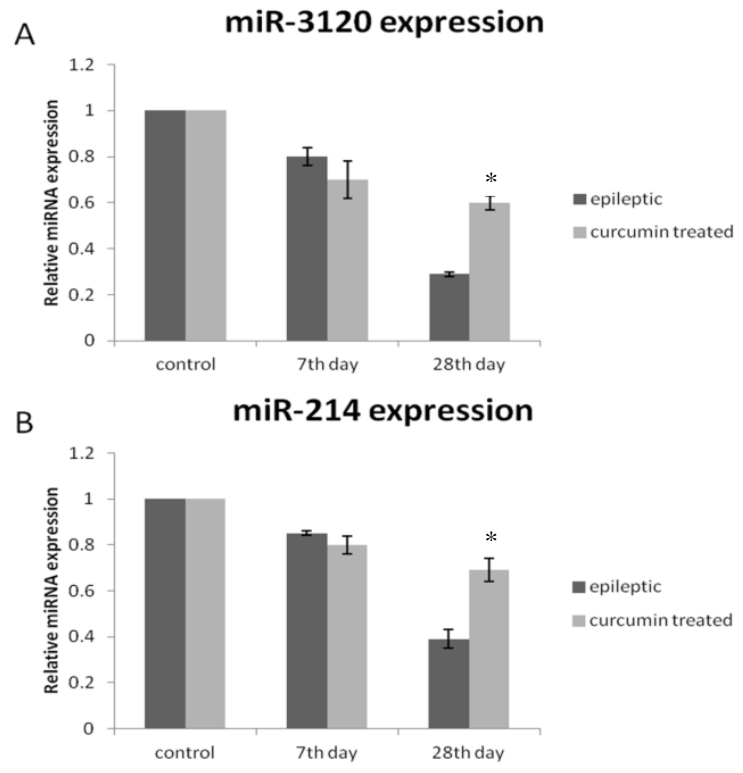

Fig 4.Effect of curcumin treatment for 7days and 28 days on miR-3120 (A) miR-214 (B) expression in the cortex of iron induced epileptic rats. Each bar represents the mean \pm SD of $n=4$ rats. Statistical comparisons of each treatment group with their respective epileptic controls cases. Increase in expression levels after curcumin treatment (28 day) is evident. $(\mathrm{n}=3, \mathrm{p}<0.05$ (ANOVA)) 


\section{Expression of Pten gene in iron-induced epilepsy:-}

Expression levels of Pten gene mRNA were measured at days 7 and 28 after induction of epilepsy. Pten gene expression levels increased with the progression of electrographic seizure activity $\left(\mathrm{F}_{2,8}=44, \mathrm{p}<0.01\right)$ (Fig. 5).

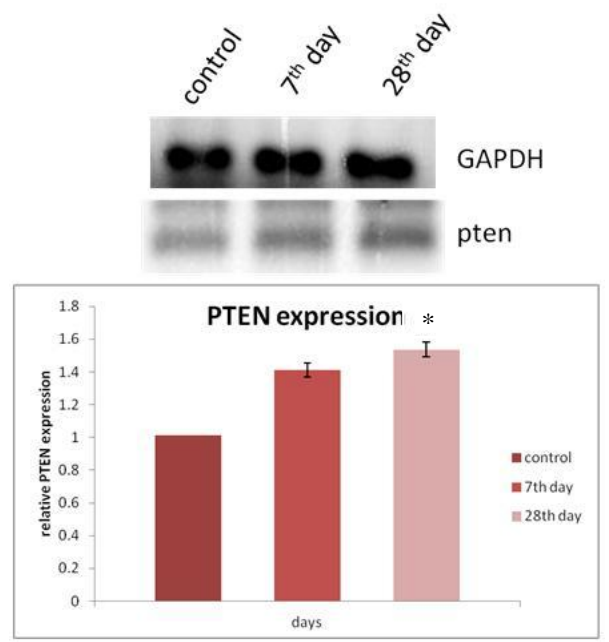

Fig 5. Relative Pten gene expression in rat cortex of iron-induced epileptic rats measured by semi qPCR. Each bar represents the mean \pm SEM levels of Pten gene mRNA. Statistical comparison of values at day 7 and 28 of epileptogenesis are with the control. Electrophoresis bands (agarose gel) of RT-PCR products corresponding to mRNA expression are presented above the $\operatorname{bars}(n=3, p<0.01($ ANOVA)) Increase in Pten gene expression with epileptogenesis, is evidnet

\section{Effect of curcumin on Pten gene expression in iron-induced epilepsy:-}

Curcumin treatment significantly decreased the expression of Pten gene (mRNA) in iron-induced epileptic rats. Seven days treatment resulted in insignificant (Fig. 6) decrease. However, 28 days treatment produced a significant decline in the expression ( $\mathrm{p}<0.01$ ) and restored the level of expression near to the control level. Thus, curcumin treatment prevented the epileptogenesis-associated rise in Pten gene expression (Fig. 7)

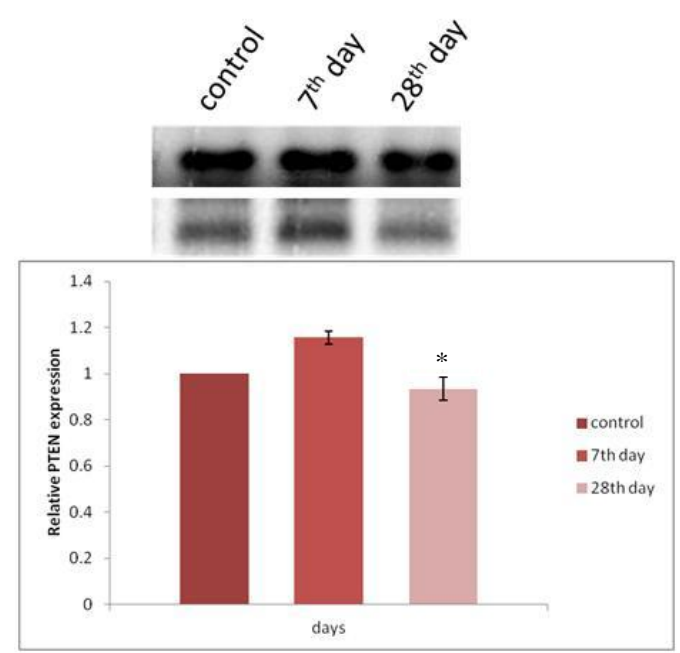

Fig 6. Effect of curcumin on relative PTEN gene expression in rat cortex of iron-induced epileptic rats measures by semi qPCR. Each bar represents the mean \pm SEM of levels of Pten gene mRNA. Statistical comparison of values at day 7 and $28^{\text {th }}$ of epleptogenesis are with the control. Electrophoresis bands (agarose gel) of RT-PCR products corresponding to mRNA expression are presented above the bars $n=3, p<0.01$ (ANOVA)) Decrease in levels of expression (comparison of values at day 7 day 28 with those of Fig- 7 are evident. 


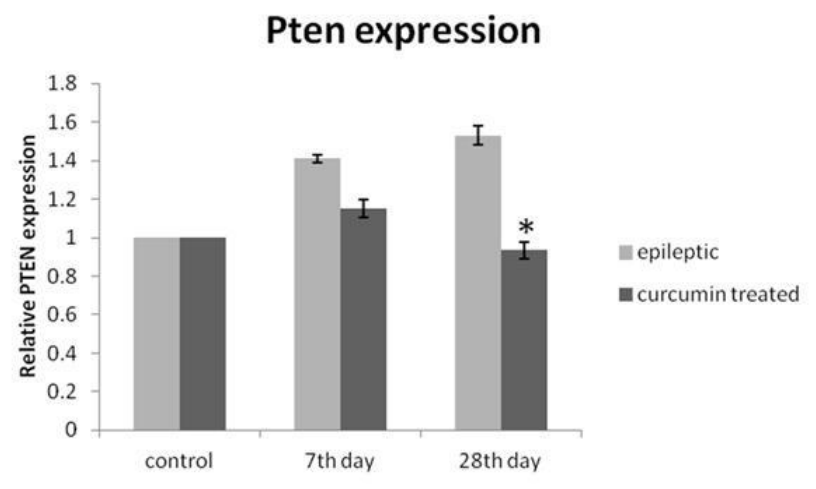

Fig 7.Effect of curcumin treatment for 7days and 28 days on Pten gene expression ( levels of Pten gene mRNA) in the cortex of iron induced epileptic rats. Each bar represents the mean $\pm S D$ of $n=4$ rats. Statistical comparisons of each treatment group with their respective epileptic controls cases. Decrease in expression levels after curcumin treatment (28 day) are evident. $(\mathrm{n}=3, \mathrm{p}<0.05$ (ANOVA))

\section{Discussion:-}

Many miRNAs have been indicated to be involved in the pathogenesis of epilepsy ( $\mathrm{Li}$ et al., 2014). For example, miR-124, miR-134, miR-196 appear to be involved in glioma- related seizures, miR-146a in temporal lobe epilepsy (TLE) and in human TLE, miR-34a and miR-210 in pilocarpine-induced status epilepticus, miR-134 in status epilepticus in epileptic rats (Dogini et al., 2015; Kretschmann et al., 2015; Chen et al., 2016). miRNAs 23 a/b and let-7e also appears to be involved in TLE (Song et al., 2011). miR-219 is involved in kainic-acid induced seizures, as well as in TLE (Zheng et al., 2016). miR-214 was also found to be down-regulated in epilepsy patients.

The data obtained in the present study showed that the levels of miR-3120 and miR-214 decreased in the cortex of iron-induced epileptic rats suggesting that these miRNAs may also be involved in the pathogenesis of epilepsies. Silencing of miRNA (by antagomir) such as miR-219 has been found to induce seizure like EEG in normal mice (Zheng et al., 2016). Therefore, decreased levels of miR-3120 and miR-214 in the cortex of iron-induced epileptic rats are indicative of the involvement of these miRNAs in iron-induced epileptogenesis. Silencing of miR-219 also resulted in increased NMDA receptor levels indicating involvement of glutamatergic mechanisms in epileptogenesis. In iron-induced epileptogenesis, upregulation of NMDA receptors, down-regulation of glutamate transporters and elevation of extracellular glutamate levels occur in iron-induced epileptogenesis (Engström et al., 2001; Mishra et al., 2013). Thus, downregulation of miR-3120 and miR-214 may be involved in the induction of glutamatergic mechanism.

The present data further showed that Pten gene expression was augmented in iron -induced epileptogenesis. Disruption of Pten gene is known to causes seizures (Backman et al., 2001). Thus the observation of increased Pten expression during epileptogenesis seems rather unexpected. Increased expression of Pten, however, could be a consequence of the depression of miR-3120 and miR-214 expressions, since these two miRNAs are known to target Pten gene (Scott et al., 2012).

Curcumin was reported to be an antiepileptic agent (Jyoti et al., 2009). In the present experiments also, curcumin was found to be an antiepileptic, as curcumin treatment of epileptic rats clearly suppressed the electrographic seizure activity. The present data further showed that curcumin stimulated the expression of miR-214 and miR-3120, and reduced the expression of Pten gene in iron-induced epileptogenesis showing that the antiepileptic action of curcumin is mediated by elevation of miR-3120, miR-214 and down-regulation of Pten gene. Curcumin's antiseizure action is also supported by the finding that it inhibits mTOR signalling (Meng et al., 2013). Thus curcumin's antiepileptic action involved elevation of miR-3120 and miR-214 and may involve inhibition of mTOR signalling. 
In summary, the present results demonstrate that iron-induced epileptogenesis is mediated by suppression of miR3120 and miR-214 expression, and curcumin's seizure-suppressive effect involves elevation of miR-3120 and miR214 expressions by curcumin. Curcumin treatment also countered epileptogenesis-associated alteration in Pten gene expression.

\section{Acknowledgement:-}

Author PK is thankful to UGC for SRF fellowship, ICMR, DST PURSE and CSIR for providing financial support to carry out this work.

\section{References:-}

1. Aronica, E., Gorter, J.A., 2007. Gene expression profile in temporal lobe epilepsy. The Neuroscientist 13, 100108.

2. Backman, S.A., Stambolic, V., Suzuki, A., Haight, J., Elia, A., Pretorius, J., Tsao, M.-S., Shannon, P., Bolon, B., Ivy, G.O., 2001. Deletion of Pten in mouse brain causes seizures, ataxia and defects in soma size resembling Lhermitte-Duclos disease. Nature Genet. 29, 396-403.

3. Cao, X., Yeo, G., Muotri, A.R., Kuwabara, T., Gage, F.H., 2006. Noncoding RNAs in the mammalian central nervous system. Annu. Rev. Neurosci. 29, 77-103.

4. Cole, G.M., Teter, B., Frautschy, S.A., 2007. Neuroprotective effects of curcumin, The molecular targets and therapeutic uses of curcumin in health and disease. Springer, pp. 197-212.

5. Chen, L., Zheng, H., Zhang, S., 2016. Involvement of upregulation of miR-210 in a rat epilepsy model. Neuropsychiatr Dis Treat. 12, 1731

6. Dogini, D.B., Avansini, S.H., Vieira, A.S., Lopes-Cendes, I., 2015. MicroRNA regulation and dysregulation in epilepsy. Regulatory RNAs in the Nervous System. 7, 172

7. Engström, E.R., Hillered, L., Flink, R., Kihlström, L., Lindquist, C., Nie, J.X., Olsson, Y., Hans, C., 2001. Extracellular amino acid levels measured with intracerebral microdialysis in the model of posttraumatic epilepsy induced by intracortical iron injection. Epilepsy Res. 43, 135-144.

8. Harraz, M.M., Eacker, S.M., Wang, X., Dawson, T.M., Dawson, V.L., 2012. MicroRNA-223 is neuroprotective by targeting glutamate receptors. Proc. Natl. Acad. Sci. U.S.A. 109, 18962-18967.

9. Jyoti, A., Sethi, P., Sharma, D., 2009. Curcumin protects against electrobehavioral progression of seizures in the iron-induced experimental model of epileptogenesis. Epilepsy Behav. 14, 300-308.

10. Kawashima, H., Numakawa, T., Kumamaru, E., Adachi, N., Mizuno, H., Ninomiya, M., Kunugi, H., Hashido, K., 2010. Glucocorticoid attenuates brain-derived neurotrophic factor-dependent upregulation of glutamate receptors via the suppression of microRNA-132 expression. Neuroscience 165, 1301-1311.

11. Kosik, K.S., 2006. The neuronal microRNA system. Nature Reviews Neuroscience 7, 911-920.

12. Kretschmann, A., Danis, B., Andonovic, L., Abnaof, K., van Rikxoort, M., Siegel, F., Mazzuferi, M., Godard, P., Hanon, E., Fr ̃̃ Thlich, H., 2015. Different microRNA profiles in chronic epilepsy versus acute seizure mouse models. J. Mol. Neurosci. 55, 466-479

13. Li, M.-M., Li, X.-M., Zheng, X.-P., Yu, J.-T., Tan, L., 2014. MicroRNAs dysregulation in epilepsy. Brain Res. 1584, 94-104.

14. Meng, X.-F., Yu, J.-T., Song, J.-H., Chi, S., Tan, L., 2013. Role of the mTOR signaling pathway in epilepsy. J. Neurol. Sci. 332, 4-15.

15. Mishra, M., Singh, R., Mukherjee, S., Sharma, D., 2013. Dehydroepiandrosterone's antiepileptic action in $\mathrm{FeCl}_{3}$-induced epileptogenesis involves upregulation of glutamate transporters. Epilepsy Res. 106, 83-91.

16. Morel, L., Regan, M., Higashimori, H., Ng, S.K., Esau, C., Vidensky, S., Rothstein, J., Yang, Y., 2013. Neuronal exosomal miRNA-dependent translational regulation of astroglial glutamate transporter GLT1. J. Biol. Chem. 288, 7105-7116.

17. Noor, N.A., Ezz, H.S.A., Faraag, A.R., Khadrawy, Y.A., 2012. Evaluation of the antiepileptic effect of curcumin and Nigella sativa oil in the pilocarpine model of epilepsy in comparison with valproate. Epilepsy Behav. 24, 199-206.

18. Papadopoulos, G.L., Alexiou, P., Maragkakis, M., Reczko, M., Hatzigeorgiou, A.G., 2009. DIANA-mirPath: Integrating human and mouse microRNAs in pathways. Bioinformatics 25, 1991-1993.

19. Rahal, O.M., Simmen, R.C., 2010. PTEN and p53 cross-regulation induced by soy isoflavone genistein promotes mammary epithelial cell cycle arrest and lobuloalveolar differentiation. Carcinogenesis 31, 14911500 . 
20. Satoskar, R., Shah, S., Shenoy, S., 1986. Evaluation of anti-inflammatory property of curcumin (diferuloylmethane) in patients with postoperative inflammation. Int J Clin Pharmacol Ther Toxicol 24, 651.

21. Scott, H., Howarth, J., Lee, Y.B., Wong, L.-F., Bantounas, I., Phylactou, L., Verkade, P., Uney, J.B., 2012. MiR-3120 is a mirror microRNA that targets heat shock cognate protein 70 and auxilin messenger RNAs and regulates clathrin vesicle uncoating. J. Biol. Chem. 287, 14726-14733.

22. Sinha, M., Ghose, J., Bhattarcharyya, N.P., 2011. Micro RNA-214,-150,-146a and-125b target Huntingtin gene. RNA Biol. 8, 1005-1021.

23. Song, Y.-j., Tian, X.-b., Zhang, S., Zhang, Y.-x., Li, X., Li, D., Cheng, Y., Zhang, J.-n., Kang, C.-s., Zhao, W., 2011. Temporal lobe epilepsy induces differential expression of hippocampal miRNAs including let-7e and miR-23a/b. Brain Res. 1387, 134-140.

24. Strimpakos, A.S., Sharma, R.A., 2008. Curcumin: preventive and therapeutic properties in laboratory studies and clinical trials. Antioxid. Redox Signal. 10, 511-546.

25. Sun, M., Estrov, Z., Ji, Y., Coombes, K.R., Harris, D.H., Kurzrock, R., 2008. Curcumin (diferuloylmethane) alters the expression profiles of microRNAs in human pancreatic cancer cells. Mol Cancer Ther. 7, 464-473.

26. Ueda, Y., Tokumaru, J., Yokoyama, H., Nakajima, A., Mitsuyama, Y., Ohya-Nishiguchi, H., Kamada, H., Willmore, L.J., 2001. Collapse of extracellular glutamate regulation during epileptogenesis: down-regulation and functional failure of glutamate transporter function in rats with chronic seizures induced by kainic acid. J. Neurochem. 76, 892-900.

27. Ueda, Y., Willmore, L.J., 2000. Sequential changes in glutamate transporter protein levels during Fe 3+-induced epileptogenesis. Epilepsy Res. 39, 201-209.

28. Wang, J., Zhou, M., Wang, X., Yang, X., Wang, M., Zhang, C., Zhou, S., Tang, N., 2014. Impact of ketamine on learning and memory function, neuronal apoptosis and its potential association with miR-214 and PTEN in adolescent rats. PloS one 9, e99855.

29. Willmore, L.J., 1990. Post-Traumatic Epilepsy: Cellular mechanisms and implications for treatment. Epilepsia 31, S67-S73.

30. Willmore, L.J., Sypert, G.W., Munson, J.B., 1978. Recurrent seizures induced by cortical iron injection: a model of posttraumatic epilepsy. Ann. Neurol 4, 329-336.

31. Yang, H., Kong, W., He, L., Zhao, J.-J., O'Donnell, J.D., Wang, J., Wenham, R.M., Coppola, D., Kruk, P.A., Nicosia, S.V., 2008. MicroRNA expression profiling in human ovarian cancer: miR-214 induces cell survival and cisplatin resistance by targeting PTEN. Cancer Res. 68, 425-433.

32. Zeng, L.-H., Rensing, N.R., Wong, M., 2009. The mammalian target of rapamycin signaling pathway mediates epileptogenesis in a model of temporal lobe epilepsy. The J. Neurosc. 29, 6964-6972.

33. Zheng, H., Tang, R., Yao, Y., Ji, Z., Cao, Y., Liu, Z., Peng, F., Wang, W., Can, D., Xing, H., 2016. MiR-219 protects against seizure in the kainic acid model of epilepsy. Mol. Neurobiol. 53, 1-7. 